\title{
Erratum to: Attenuation of $A \beta$ toxicity by promotion of mitochondrial fusion in neuroblastoma cells by liquiritigenin
}

Doo Sin Jo ${ }^{1}$ - Dong Woon Shin ${ }^{1}$ So Jung Park ${ }^{1} \cdot$ Ji-Eun Bae $^{1} \cdot$ Joon Bum Kim $^{1}$

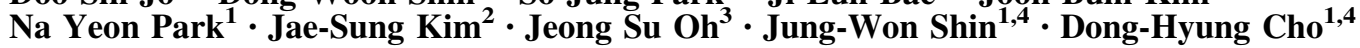

Published online: 11 September 2016

(C) The Pharmaceutical Society of Korea 2016

Erratum to: Arch. Pharm. Res. (2016)

39:1137-1143

DOI 10.1007/s12272-016-0780-2

It is note that this article initially has been published as a review article, however, it is a research article. We here correct this paper as an original research article. We apologize for this confusion or ambiguity caused regarding format of our article.
The online version of the original article can be found under doi:10.1007/s12272-016-0780-2.

\footnotetext{
Jung-Won Shin

shinarago@khu.ac.kr

$\bowtie$ Dong-Hyung Cho

dhcho@khu.ac.kr

Doo Sin Jo

doosinjo@gmail.com

Dong Woon Shin

shin901023@naver.com

So Jung Park

shzz34@hanmail.net

Ji-Eun Bae

loveg730@hotmail.com

Joon Bum Kim

kss3213@naver.com

Na Yeon Park

yeonie5613@gmail.com

Jae-Sung Kim

jaesung@kirams.re.kr

Jeong Su Oh

ohjs@skku.edu
}

1 Graduate School of East-West Medical Science, Kyung Hee University, Yongin-Si, Gyeonggi-Do 17104, Republic of Korea

2 Division of Radiation Cancer Research, Korea Institute of Radiological and Medical Science, Seoul 01812, Republic of Korea

3 Department of Genetic Engineering, College of Biotechnology and Bioengineering, Sungkyunkwan University, Suwon 16356, Republic of Korea

4 Graduate School of East-West Medical Science, Kyung Hee University, 1732, Deogyeong-daero, Giheung-gu, Yongin-Si, Gyeonggi-Do 17104, Republic of Korea 